\title{
Association between waking EEG and cognitive event-related potentials in patients with obstructive sleep apneas
}

\author{
Andrée-Ann Baril, B.Sc candidate, ${ }^{1,2}$ Katia Gagnon, B.Sc. ${ }^{1,3}$ Jean-François Gagnon, \\ Ph.D., ${ }^{1,3}$ Jacques Montplaisir, M.D., Ph.D. ${ }^{1,4}$ Nadia Gosselin, Ph.D. ${ }^{1,4^{*}}$
}

1. Center for Advanced Research in Sleep Medicine, Hôpital du Sacré-Cœur de Montréal, Montreal, Qc, Canada

2. Faculty of Medicine, University of Montreal, Montreal, Qc, Canada

3. Department of Psychology, Université du Québec à Montréal, Qc, Canada

4. Department of Psychiatry, University of Montreal, Montreal, Qc, Canada

Submitted to: Sleep Medicine (brief communication)

Date: October $12^{\text {th }}, 2012$

Short Title: EEG and ERP in OSA

Address for correspondence:

Nadia Gosselin, Ph.D.

Center for Advanced Research in Sleep Medicine

Hôpital du Sacré-Cœur de Montréal

5400 boul. Gouin Ouest, local E-0330

Montréal, Québec

H4J 1 C5

Canada

Tel: 514-338-2222 ext. 7717

Fax: 514-338-3893

nadia.gosselin@umontreal.ca

Keywords: Obstructive sleep apnea; Event-related potentials; Quantitative electroencephalography; Attention; Vigilance; Cognition; P300; P3a 


\begin{abstract}
Objective: Abnormal event-related potentials (ERP) and slowing of the waking electroencephalographic (EEG) activity have been reported in patients with obstructive sleep apneas (OSA). This study aimed at evaluating whether an association exists between the severity of ERP abnormalities and EEG slowing in order to better understand cerebral dysfunction in OSA.

Methods: Twelve OSA patients and 12 age-matched controls underwent an overnight polysomnographic recording, an EEG recording of 10 minutes of wakefulness and an auditory ERP protocol known to specifically measure attention. Amplitude and latency of each ERP component were measured as well as the spectral power in each frequency band of the waking EEG. Pearson's correlations were used to measure associations between ERP characteristics and EEG spectral power in OSA patients and control subjects.
\end{abstract}

Results: A positive correlation between the late P300 amplitude and theta power in occipital region was observed in OSA subjects $(\mathrm{p}<0.01)$. A positive correlation was also found between $\mathrm{P} 3 \mathrm{a}$ amplitude and beta 1 power in central region in OSA subjects $(\mathrm{p}<0.01)$. No correlation was observed for control subjects.

Conclusions: ERP abnormalities observed in an attention task are associated with a slowing of the waking EEG recorded at rest in OSA. 


\section{Introduction}

Obstructive sleep apnea (OSA) is a sleep-related breathing disorder characterized by repetitive pauses (apneas) or reductions in airflow amplitude (hypopneas) attributed to the collapsibility of the upper airway during sleep. OSA causes sleep disruption and intermittent nocturnal hypoxemia, which can lead to daytime sleepiness, cognitive deficits, and cerebral dysfunctions [1].

Previous studies using event-related-potentials (ERP) have consistently shown delayed P300 latencies and/or changes in P300 amplitudes suggesting attention deficits in OSA [2]. In previous studies performed by our group, multiple ERP components were investigated, i.e. N1, mismatch negativity (MNN), P3a, P300, and reorienting negativity $(\mathrm{RON})$, in order to assess different stages of information processing (i.e. pre-attentional and attentional processes) in OSA [3-4]. The major finding was that OSA patients showed a wide and prolonged P300 as well as a reduction in P3a amplitude, suggesting normal automatic detection of stimulus but abnormal attentional processes.

Quantitative electroencephalography (qEEG) is another well-established method for evaluating brain functions. Quantitative EEG studies in OSA patients demonstrated a significant EEG slowing during wakefulness over all cortical regions, suggesting lower levels of vigilance and/or a general brain dysfunction [5-7]. However, to date, the relationship between waking EEG anomalies observed in a resting condition and ERP changes observed during a cognitive task has not been investigated in OSA patients. Therefore, the aim of the present study was to evaluate whether the severity of ERP 
abnormalities correlated with the waking EEG slowing, in order to better understand cerebral dysfunctions associated with OSA. We hypothesized that P300 latency and amplitude would be the variables most correlated with EEG slowing, as defined by an increased power in slow frequency bands and a decreased power in fast frequency bands, since the P300 component is generally considered an index of general cognitive functioning and/or attentional resources [8-10].

\section{Methods}

\section{Subjects}

Subjects included in the present study have been described in a previous paper [3].

Briefly, the study groups consisted of 12 OSA patients $(9 \mathrm{M}, 47.9 \pm 13.7$ years old, 12.5 \pm 3.3 years of education) and 12 healthy control subjects $(11 \mathrm{M}, 44.4 \pm 9.5$ years old, $14.2 \pm 2.8$ years of education). None of the subjects included had auditory deficit, unstable high blood pressure, neurological or psychiatric diseases or a pulmonary disease. Subjects who use drugs known to affect sleep or daytime sleepiness were excluded. Each participant was informed of the research protocol and gave written consent before the beginning of the study. A University-Hospital ethics committee approved the protocol.

\section{Polysomnographic recording}

Participants underwent a standard all-night polysomnographic evaluation with 16 surface EEG electrodes referred to linked earlobes, electrooculogram, chin electromyogram, thoracoabdominal plethysmograph, oral/nasal canula and transcutaneous finger pulse 
oximeter. Sleep was scored according to standard criteria [11]. OSA patients had an apnea-hypopnea index (AHI) higher than 10 per hour of sleep (group average: $51.2 \pm$ 23.9) and control subjects had an AHI under 5 (group average: $1.2 \pm 1.4$ ).

\section{Waking EEG recording and $q E E G$ analysis}

Ten minutes of waking EEG with eyes closed were recorded 30 minutes after morning awakening. Subjects were asked to open their eyes periodically and constant monitoring was done to insure that subjects were not falling asleep. On average, 104 seconds $( \pm 21)$ without artifact were selected for each subject, varying from 52 to $124 \mathrm{~s}$. EEG spectral power was determined on 4-s epochs using fast-Fourier transforms and EEG segments were analyzed with cosine tapering at a $0.25 \mathrm{~Hz}$ resolution. The analyzed frequency bands were delta $(0.5-3.75 \mathrm{~Hz})$, theta $(4-7.75 \mathrm{~Hz})$, alpha $(8-12.75 \mathrm{~Hz})$, beta $1(13-21.75$ $\mathrm{Hz})$ and beta $2(22-31.75 \mathrm{~Hz})$. The data were analyzed using a software package (Harmonie Stellate Systems@ $@$, Montreal, Canada) on 4 electrodes: F3, C3, P3 and O1. The absolute and relative power for each frequency band was calculated for each region and subject. The slow to fast frequency ratio was calculated using [(Delta + Theta)/(Alpha + Beta $1+$ Beta 2) $]$.

\section{ERP recording and analysis}

The auditory ERP protocol and data analyses have been described previously [3]. The ERP task was done between 8:00 and 9:30 AM, after polysomnographic and waking EEG recordings. Briefly, we presented a dichotic sequence of short (50\%) and long (50\%) 
tones and, for these two types of tones, $90 \%$ were standard $(1000 \mathrm{~Hz})$ and $10 \%$ were deviant $(750$ or $1250 \mathrm{~Hz})$ tones. Subjects were instructed to distinguish short from long tones by pressing buttons. Amplitude and latency of ERP components (i.e. N1, MMN, P3a, P300, and RON) were analyzed on F3, C3, P3 and O1 electrodes.

\section{Statistical analyses}

Between-group differences on demographic, polysomnographic and ERP variables have been presented in our previous study [3], and were therefore not reanalyzed in the present study. Group differences on qEEG were analyzed using Group (OSA; Control) by Electrode (F3, C3, P3, O1) ANOVAs for each frequency band. Pearson's correlation coefficients were used to measure relationships between ERP characteristics (amplitude and latency) and qEEG (spectral power) in OSA patients and control subjects separately. Analyses were considered significant at $\mathrm{p}<0.05$. STASTICA 6.0 was used for all statistical analyses.

\section{Results}

\section{Group differences on $q E E G$}

No between-group differences were observed for relative or absolute EEG power in any frequency band. Moreover, no group difference was found for the slow to fast frequency ratio. 


\section{Relationship between ERP and qEEG variables}

Figure 1 shows the correlations between ERP and qEEG variables. A significant correlation was found between late P300 amplitude and absolute and relative theta power for OSA patients on $\mathrm{O} 1$ ( $\mathrm{r}=0.75$ and 0.80 respectively, $p<0.01)$, where higher $\mathrm{P} 300$ amplitudes elicited by standard stimuli were associated with higher theta power. A significant correlation was also observed between P3a amplitude and absolute beta 1

power on $\mathrm{C} 3$ in OSA patients $(\mathrm{r}=0.78, p<0.01)$; higher $\mathrm{P} 3 \mathrm{a}$ amplitudes for deviant stimuli (observed on the subtraction between deviant and standards waveforms) were associated with lower beta 1 power. These correlations were not observed in the control group. No other correlations were found between amplitude and latency of ERP components and qEEG.

\section{Discussion}

The aim of the present study was to correlate ERP abnormalities with waking EEG slowing in order to better understand cerebral dysfunctions in OSA patients. This study represents the first attempt to characterize the relationship between abnormal EEG activities recorded in two different conditions, i.e. at rest and during an attention task, in OSA. We found that higher late P300 amplitudes measured during our attention task were associated with increased EEG theta power during a rest condition. Moreover, decreased P3a amplitudes were associated with decreased beta 1 power.

In our previous study [3], a prolonged and sustained P300 wave was observed in OSA, 
probably reflecting increased attentional resources allocated to the task compared to control subjects. In the present study, the amplitude of this sustained P300 component was found to be associated with an increase in waking EEG theta power in the occipital region. Since P300 is generally associated with attention processes in tasks requiring target detection and classification [9-10] and increased theta power represents impaired vigilance or sleepiness [12], our results suggest that the P300 changes observed in OSA is associated with vigilance decrement. The protocol used in this study does not allow verifying whether vigilance impairment are the cause of abnormal P300 during the auditory attentional task or whether changes in P300 and theta power are two independent manifestations of a more general cerebral dysfunction in OSA. However, the vigilance and attention deficits observed in the present study probably interfere with other cognitive processes, since they play a pivotal role in other cognitive domains, such as executive functions and episodic memory [13-14]. Interestingly, the correlation between the P300 component and theta power was specific to OSA and was not observed in the control group. The lack of variability in P300 and theta power observed in the control group may explain the absence of relationship between these two variables.

A significant correlation between P3a amplitude and beta 1 power was also found in the present study, where decreased $\mathrm{P} 3 \mathrm{a}$ were associated with reduced beta 1 power. As for the P300, changes in P3a amplitude were documented in our previous study in OSA patients [4]. P3a is known to represent orientation of attention toward unexpected stimuli, while beta activity is considered to be an index of brain activation and alertness in response to attention requirements [15-16]. Interestingly, P3a amplitude was not 
correlated with slow EEG frequency, such as delta and theta frequency bands, that are associated with vigilance level, which suggest that P3a anomalies are not caused by a general vigilance decrement during the attentional task.

In conclusion, anomalies observed in waking EEG recorded at rest are associated with changes in ERP recorded during attentional task in OSA. Further studies investigating the causal role of altered waking EEG in cognitive dysfunctions, such as those measured with ERP and neuropsychological assessment will be needed in this population. Moreover, the possible differential effect of OSA treatment on EEG and ERP should be investigated.

\section{Acknowledgments:}

The authors thank Annie Mathieu, Ph.D., for patient recruitment and testing. The authors also thank Hélène Blais, B.A., and Jean Paquet, Ph.D, for helping in analyses and statistics and Dominique Petit, Ph.D, and Catherine Duclos, B.A., for reviewing the manuscript.

\section{Sources of financial support:}

This study was supported by the Canadian Institutes of Health Research (CIHR operating

grant to N.G., J.F.G. and J.M.), by the Department of Psychiatry of the University of Montreal (COPSE studentship to A.A.B.), and by the Fonds pour la Recherche du Québec-Nature et technologie (studentship to K.G.). 


\section{Figure legends}

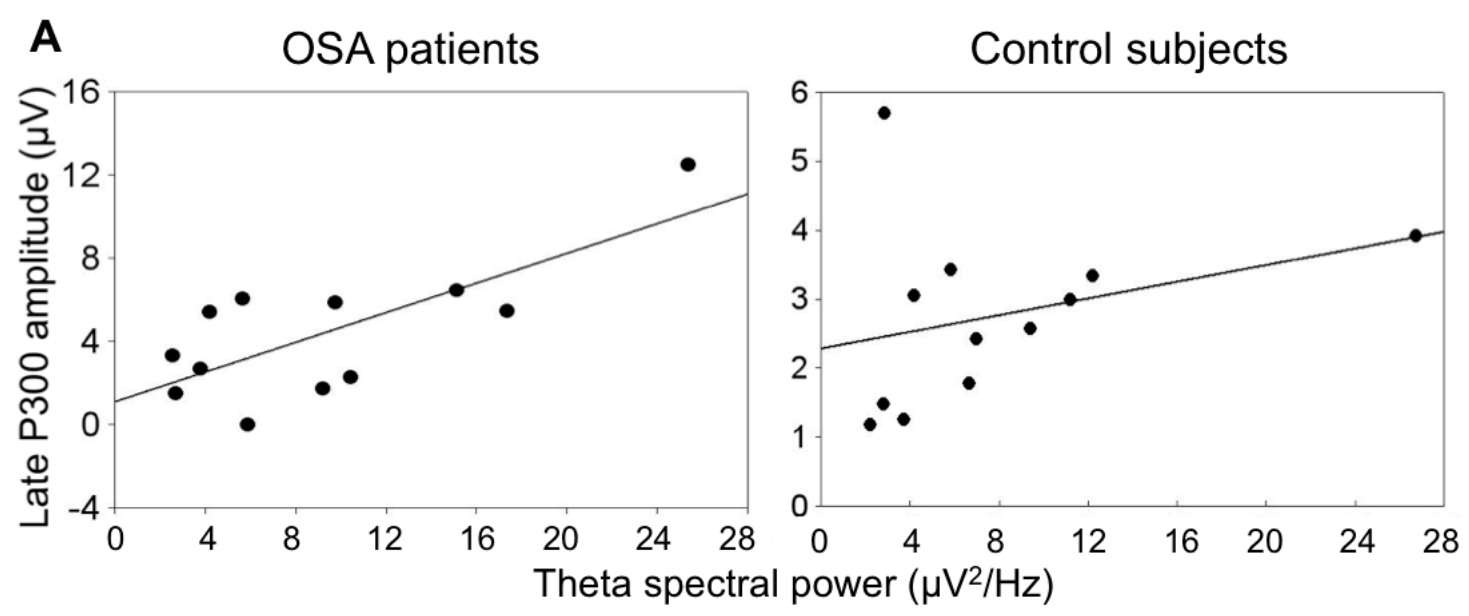

B

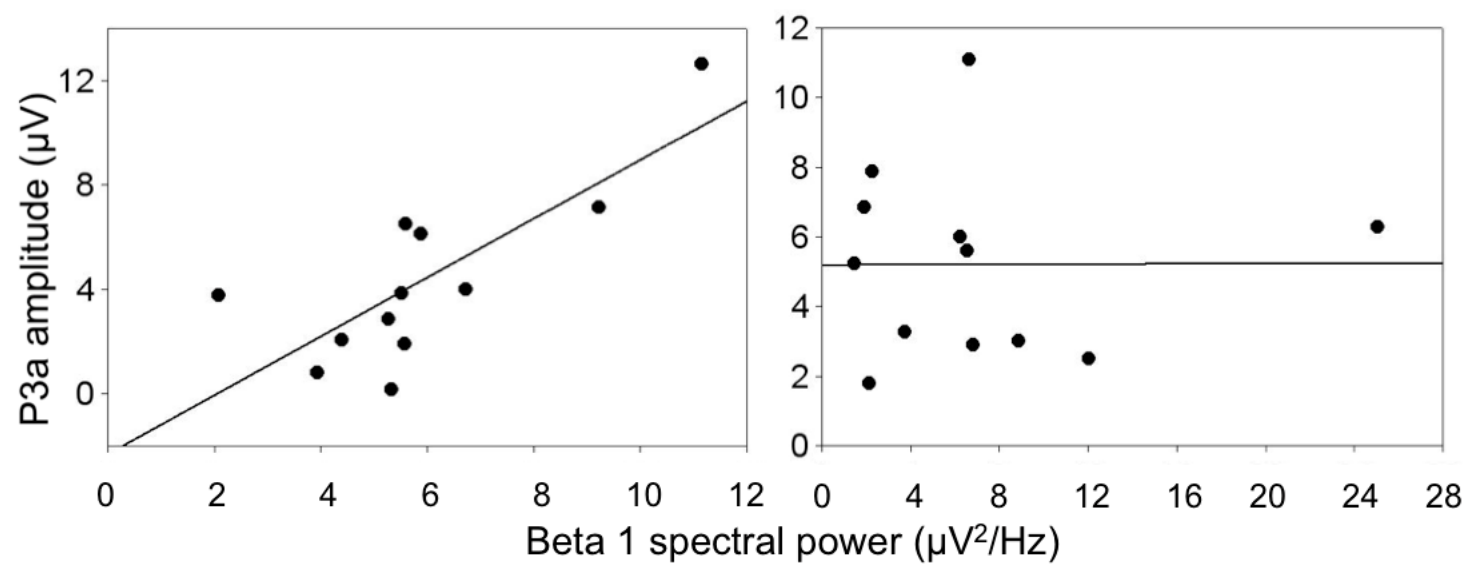

Figure 1. Correlation between late P300 amplitude and absolute theta power (A) and between P3a amplitude and absolute beta 1 power (B) in OSA subjects (left) and control subjects (right). (n.s., non significant)

\section{References}

[1] Beebe DW, Gozal D. Obstructive sleep apnea and the prefrontal cortex: towards a comprehensive model linking nocturnal upper airway obstruction to daytime cognitive and behavioral deficits. J Sleep Res 2002;11:1-16.

[2] Raggi A, Ferri R. Cognitive evoked potentials in obstructive sleep apnea 
syndrome: a review of the literature. Rev Neurosci 2012;23:311-323.

[3] Gosselin N, Mathieu A, Mazza S, Petit D, Malo J, Montplaisir J. Attentional deficits in patients with obstructive sleep apnea syndrome: An event-related potential study. Clin Neurophysiol 2006;117:2228-2235.

[4] Gosselin N, Mathieu A, Mazza S, Decary A, Malo J, Montplaisir J. Deficits in involuntary attention switching in obstructive sleep apnea syndrome. Neurosci Lett 2006;408:73-78.

[5] Mathieu A, Mazza S, Petit D, Decary A, Massicotte-Marquez J, Malo J, Montplaisir J. Does age worsen EEG slowing and attention deficits in obstructive sleep apnea syndrome? Clin Neurophysiol 2007;118:1538-1544.

[6] Xiromeritis A, Hatziefthimiou A, Hadjigeorgiou G, Gourgoulianis K, Anagnostopoulou D, Angelopoulos N. Quantitative spectral analysis of vigilance EEG in patients with obstructive sleep apnoea syndrome. Sleep Breath 2011;15:121-128.

[7] Morisson F, Lavigne G, Petit D, Nielsen T, Malo J, Montplaisir J. Spectral analysis of wakefulness and REM sleep EEG in patients with sleep apnoea syndrome. Eur Respir J 1998;11:1135-1140.

[8] Picton TW. The P300 wave of the human event-related potential. J Clin Neurophysiol 1992;9:456-479.

[9] Kramer AF, Strayer DL. Assessing the development of automatic processing: an application of dual-task and event-related brain potential methodologies. Bio Psychol 1988;26:231-267.

[10] Wickens C, Kramer A, Vanasse L, Donchin E. Performance of concurrent 
tasks: a psychophysiological analysis of the reciprocity of informationprocessing resources. Science 1983;221:1080-1082.

[11] Rechtschaffen A, Kales A. A manual of standardized terminology, techniques and scoring system for sleep stages of human subjects. Los Angeles: Brain Information Service/Brain Research Institute, 1968.

[12] De Gennero L, Marzano C, Veniero D, Moroni F, Fratello F, Curcio G, et al. Neurophysiological correlates of sleepiness: A combined TMS and EEG study. Neuroimage 2007;36:1277-1287.

[13] Mazza S, Pepin JL, Naegele B, Plante J, Deschaux C, Levy P. Most obstructive sleep apnoea patients exhibit vigilance and attention deficits on an extended battery of tests. Eur Respir J 2005;25:70-80.

[14] Verstraeten E, Cluydts R. Executive control of attention in sleep apnea patients: theoretical concepts and methodological considerations. Sleep Med Rev 2004;8:257-267.

[15] Ray WJ, Cole HW. EEG alpha activity reflects attentional demands, and beta activity reflects emotional and cognitive processes. Science 1985;10:750-752.

[16] Kaminski J, Brzezicka A, Gola M, Wrobel A. Beta band oscillations engagement in human alertness process. Int J Psychophysiol 2012;85:125-128. 